\title{
Análisis Morfométrico de los Reparos Anatómicos Involucrados en la Técnica Quirúrgica de Colocación de Implantes Cigomáticos
}

\author{
Morphometric Analysis of Anatomic Landmarks Involved \\ in Surgical Technique of Zygomatic Implants Placement
}

\begin{abstract}
Alves, $\mathbf{N}$.
ALVES, N. Análisis morfométrico de los reparos anatómicos involucrados en la técnica quirúrgica de colocación de implantes cigomáticos. Int. J. Morphol., 31(3):986-990, 2013.

RESUMEN: El objetivo de esta investigación fue ofrecer datos morfométricos de las estructuras óseas involucradas en la técnica quirúrgica de colocación de implantes cigomáticos, proveyendo así, informaciones que faciliten la planificación y realización de dicho procedimiento. Considerando los valores promedios obtenidos en nuestro estudio, concluimos que el mayor espesor del hueso cigomático se encuentra en la zona media del hueso. Sugerimos que el anclaje superior del implante cigomático no debe extenderse hasta el margen posterosuperior del hueso cigomático, ya que el espesor del hueso a este nivel no ofrece condiciones de adecuada oseointegración de implantes.
\end{abstract}

PALABRAS CLAVE: Implantes zigomáticos; Morfometría.

\section{INTRODUCCIÓN}

Con la pérdida de los dientes el hueso alveolar pierde su razón de existir y se reabsorbe, permaneciendo apenas resquicios del proceso alveolar sobre el hueso basal de soporte, denominados rebordes residuales (Alves \& Cândido, 2013).

Las prótesis y los implantes dentales buscan retrasar o incluso evitar este proceso de reabsorción ósea, mientras tratan de sustituir de alguna manera a los dientes originales perdidos. Pero, en algunos casos, la reabsorción ósea es tal que limita o impide la colocación de implantes inmediatos (Alves \& Cândido). Una de las áreas donde más se observa este fenómeno es en la región posterior del maxilar, considerada una de las más difíciles de rehabilitar.

Para el tratamiento de maxilares que presentan reabsorción severa se han descrito una serie de procedimientos, tales como la elevación de seno, injertos óseos onlay, injertos óseos combinados con osteotomías Lefort I. Dichos procedimientos han proporcionado un adecuado volumen óseo para la instalación de implantes (Bedrossian et al., 2002; Boyes-Varley et al., 2003; Nakai et al., 2003; Nkenke et al., 2003), sin embargo pueden ser contraindicados en ciertas situaciones. Para estos casos surgió una alternativa relativamente reciente de tratamiento, desarrollada por Bränemark y colaboradores, que son los implantes cigomáticos (BoyesVarley et al.). La técnica original descrita por Bränemark (1998) establece la colocación de un implante único en el hueso cigomático, bilateralmente, combinado con al menos dos implantes convencionales en la región anterior del maxilar (Uchida et al., 2001; Bothur et al., 2003).

Los implantes cigomáticos, son implantes en 45 grados, presentando entre 35 y $55 \mathrm{~mm}$ de longitud, que se introduce en la parte palatina del proceso alveolar, región del segundo premolar, atravesando el seno maxilar hasta anclarse en el hueso cigomático. Están indicados para situaciones de atrofia del maxilar, reabsorción ósea extensa, senos maxilares neumatizados o cuando no sea posible la instalación de implantes convencionales (Stevenson \& Austin, 2000; Vrielinck et al., 2003). Además, se han utilizado en pacientes tras maxilectomías por tumores y enfermedades que se asocian a situaciones de atrofia en el maxilar (Weischer $e t$ al., 1997; Tamura et al., 2000).

El objetivo de esta investigación es ofrecer datos morfométricos de las estructuras óseas involucradas en la técnica quirúrgica de colocación de implantes cigomáticos, proveyendo así, informaciones que faciliten la planificación y realización de dicho procedimiento. 


\section{MATERIAL Y MÉTODO}

Fueron analizados en este trabajo 153 cráneos humanos de brasileños adultos, de ambos sexos, leucodermas y melanodermas, pertenecientes al Museo de Cráneos del Departamento de Morfología de la Universidade Federal de São Paulo, UNIFESP, Brasil. De estos, 59 cráneos eran de mujeres, con edades entre 20 y 100 años (edad promedio $=$ 41,13 años), y 94 cráneos eran de hombres, con edades entre 21 y 70 anos (edad promedio $=39,64$ años).

En cada cráneo se realizaron mediciones bilaterales, utilizando para ello un calibrador tipo pie de rey digital con exactitud de $0,1 \mathrm{~mm}$. Las mediciones realizadas fueron las siguientes:

- Distancia desde el reborde alveolar a nivel del segundo premolar por su cara palatina hasta el margen posterosuperior del hueso cigomático (distancia A-B). El margen posterosuperior corresponde al punto de unión entre los procesos frontal y temporal del hueso cigomático (Fig. 1A);

- Espesor del hueso cigomático a nivel del margen posterosuperior (C) (Fig. 1B);

- Espesor del hueso cigomático en su porción media (D) (Fig. 1C). El punto medio fue determinado como el punto de intersección entre dos rectas: una tangenciando el margen superior del proceso temporal y otra tangenciando el margen anterior del proceso frontal del hueso cigomático.

Fueron analizadas las variables lado (derecho e izquierdo) y género (masculino y femenino) a través de las pruebas t-student y Mann-Whitney, según corresponde.

\section{RESULTADOS}

Los valores promedios encontrados para las distancias A-B, C y D fueron: $59,92 \mathrm{~mm}, 2,32 \mathrm{~mm}$ y $5,68 \mathrm{~mm}$ para os hombres (Tabla I) y $53,41 \mathrm{~mm}, 2,08 \mathrm{~mm}$ e $4,88 \mathrm{~mm}$ para las mujeres, respectivamente (Tabla II). Los valores mínimos, máximos y promedios para los lados derecho e izquierdo están expresos en las Tablas I (para hombres) y II (para mujeres).

Observamos que los valores promedios encontrados para los hombres fueron mayores que los encontrados para mujeres en todas las distancias.

No hubo diferencia estadística significativa en la variable lado para hombres o mujeres.

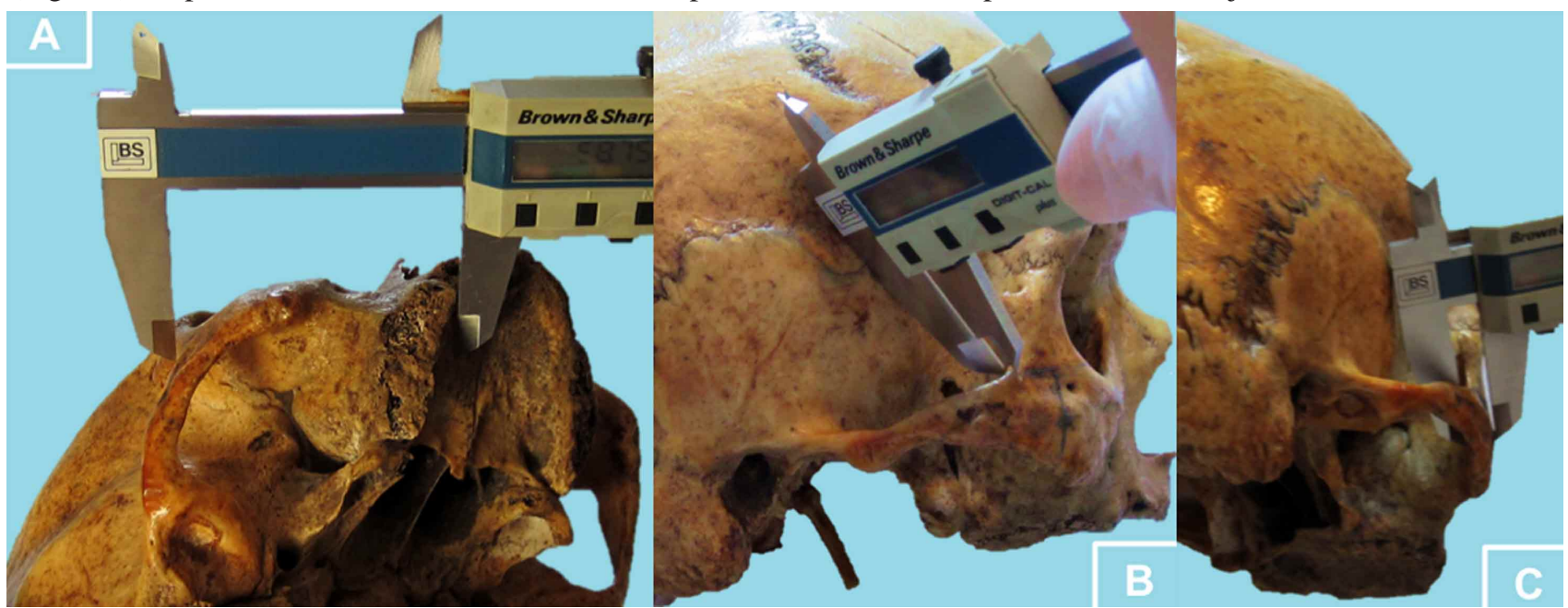

Fig. 1. Mediciones realizadas en los cráneos.

Tabla I. Valores promedios, mínimos, máximos y valor-p de las distancias encontradas para hombres (mm).

\begin{tabular}{|c|c|c|c|c|c|c|}
\hline Distancia & \multicolumn{2}{|c|}{ A-B } & \multicolumn{2}{|c|}{$\mathbf{C}$} & \multicolumn{2}{|c|}{ D } \\
\hline Lado & Derecho & Izquierdo & Derecho & Izquierdo & Derecho & Izquier do \\
\hline Promedio & 57,28 & 56,56 & 2,32 & 2,33 & 5,80 & 5,55 \\
\hline Valor-p & \multicolumn{2}{|c|}{0,34} & \multicolumn{2}{|c|}{0,95} & \multicolumn{2}{|c|}{0,15} \\
\hline Máximo & 63,39 & 64,62 & 3.77 & 4,30 & 8,81 & 8,80 \\
\hline Mínimo & 50,45 & 50,14 & 1.03 & 1,32 & 3,15 & 3,07 \\
\hline Promedio (derecho e izquierdo) & \multicolumn{2}{|c|}{56,92} & \multicolumn{2}{|c|}{2,32} & \multicolumn{2}{|c|}{5,68} \\
\hline
\end{tabular}


Tabla II. Valores promedios, mínimos, máximos y valor-p de las distancias encontradas para mujeres (mm).

\begin{tabular}{lccccccc}
\hline Distancia & \multicolumn{2}{c}{ A-B } & \multicolumn{2}{c}{ C } & \multicolumn{2}{c}{ D } \\
Lado & Derecho & Izquierdo & Derecho & Izquierdo & Derecho & Izquierdo \\
\hline Promedio & 53,57 & 53,25 & 2,10 & & 2,07 & 4,90 & 4,85 \\
Valor-p & \multicolumn{2}{c}{0,59} & & & 0,71 & & \\
Máximo & 62,49 & 60,28 & 3,07 & & 3,69 & 7,80 & 7,21 \\
Mínimo & 46,19 & 45,47 & 1,22 & 0,81 & 2,69 & 2,92 \\
Promedio (derecho e izquierdo) & \multicolumn{2}{c}{53,41} & & 2,08 & & & 4,88 \\
\hline
\end{tabular}

Tabla III. Porcentaje de implantes zigomáticos utilizados por Pi Urgell et al. (2008), de acuerdo a la longitud.

\begin{tabular}{lllllllll}
\hline Longitud & $30 \mathrm{~mm}$ & $35 \mathrm{~mm}$ & $40 \mathrm{~mm}$ & $42,5 \mathrm{~mm}$ & $45 \mathrm{~mm}$ & $47,5 \mathrm{~mm}$ & $50 \mathrm{~mm}$ & $52,5 \mathrm{~mm}$ \\
\hline$\%$ de implantes & 0 & $9,90 \%$ & $26,75 \%$ & $15,85 \%$ & $7,90 \%$ & $23,75 \%$ & $15,85 \%$ & $0 \%$ \\
\hline
\end{tabular}

Tabla IV. Porcentaje de casos encontrados para hombres y mujeres de acuerdo a la distancia A-B (mm).

\begin{tabular}{lllllllll}
\hline & $45-47,5$ & $47,5-50$ & $50-52,5$ & $52,5-55$ & $55-57,5$ & $57,5-60$ & $60-62,5$ & $62,5-65$ \\
\hline Hombres & $0 \%$ & $0 \%$ & $7,44 \%$ & $18,61 \%$ & $25,53 \%$ & $28,72 \%$ & $14,90 \%$ & $4,80 \%$ \\
Mujeres & $3,38 \%$ & $9,32 \%$ & $29,7 \%$ & $24,57 \%$ & $21,18 \%$ & $10,15 \%$ & $1,70 \%$ & $0 \%$ \\
\hline
\end{tabular}

Al analizar la variable género encontramos diferencia estadística significativa en todas las distancias para el lado derecho (A-B, p<0,0001; C, p=0,01; D, p<0,0001) y para el lado izquierdo (A-B, $\mathrm{p}<0,0001 ; \mathrm{C}, \mathrm{p}<0,005 ; \mathrm{D}$, $\mathrm{p}=0,001)$.

\section{DISCUSIÓN}

En las mediciones realizadas en nuestro estudio encontramos que el valor promedio del espesor del hueso cigomático a nivel del margen posterosuperior fue 2,32 mm en hombres y 2,08 mm en mujeres. Considerando esta misma región, González et al. (2007), analizando 25 cráneos encontraron un espesor óseo promedio de 3,18 mm. Concordamos con estos autores cuando plantean que es dudoso el real valor de un anclaje superior del implante en esta región. Según Uchida et al., el espesor del hueso cigomático, a nivel del margen posterosuperior, debería medir al menos $5,75 \mathrm{~mm}$ para poder instalar un implante cigomático en esta región. Aunque Fernández Ateca et al. (2004) afirmen que el ápice de la fijación del implante cigomático puede sobresalir 1 o 1,5 mm de la cortical del cigomático, Frodel et al. (1993), afirman que el hueso que debe rodear a un implante debe ser de al menos $1 \mathrm{~mm}$ de espesor, para que ocurra la oseointegración. Tomando en cuenta estas afirmaciones y además los valores obtenidos en nuestra investigación, podemos afirmar que el espesor del hueso cigomático a nivel del margen posterosuperior no ofrece condiciones de adecuada oseointegración de implantes colocados en este sitio.
En relación al espesor del hueso cigomático en su porción media, las mediciones realizadas en nuestro estudio nos permitieron obtener el valor promedio de 5,68 $\mathrm{mm}$ en hombres y 4,88 $\mathrm{mm}$ en mujeres. Nuestros hallazgos nos permiten concordar con González et al., cuando afirman que el mayor espesor del hueso cigomático se encuentra en la zona media del hueso. Aunque estos autores no hayan analizado género, obtuvieron un valor promedio de 5,36 mm en las mediciones realizadas en esta misma región, valor similar a los nuestros.

En nuestro estudio, los valores promedios obtenidos para las distancias desde el reborde alveolar a nivel del segundo premolar por su cara palatina hasta el margen posterosuperior del hueso cigomático (distancia A-B) fueron 56,92 mm en hombres (máximo 64,62 mm; mínimo $50,14 \mathrm{~mm}$ ) y 53,41 $\mathrm{mm}$ en mujeres (máximo 62,49 mm; mínimo 45,47 mm).

Pi Urgell et al. (2008), evaluaron la supervivencia de 101 implantes cigomáticos colocados en maxilas con características de reabsorción importantes en la cresta alveolar posterior. Estos 101 implantes Zygoma ${ }^{\circledR}$ (Nobel Biocare, Götenborg, Suecia) se utilizaron junto con 221 implantes Brånemark System ${ }^{\circledR}$ estándar (Nobel Biocare, Gotenborg, Suecia). El porcentaje de longitudes de los implantes cigomáticos utilizados, disponibles en 8 diferentes longitudes (entre 30 y 52,5 mm) se muestra en la Tabla III. Comparando estos datos con los obtenidos en nuestro trabajo (Tabla IV) observamos que $87,3 \%$ de las mujeres presentaron la distancia A-B con valores superiores a 50 mm (ma- 
yor valor utilizado por Pi-Urgell et al.), mientras que para los hombres este valor fue $100 \%$. Es importante recordar que la distancia A-B, utilizada en nuestro trabajo, corresponde al mismo trayecto recorrido por los implantes cigomáticos utilizados por Pi-Urgell et al.

Los implantes cigomáticos se encuentran disponibles en distintas longitudes, variando desde 30 hasta $62,5 \mathrm{~mm}$, dependiendo del fabricante (Boyes-Varley et al.). Considerando los valores obtenidos en nuestra investigación y las diferencias existentes entre los fabricantes, creemos que los profesionales pueden necesitar más de un sistema quirúrgico para cumplir con todas las medidas, en caso de que decidan por la colocación de un implante cigomático con el ápice llegando hasta el margen posterosuperior del hueso cigomático.

En la literatura están descritas tres técnicas quirúrgicas para la colocación de implantes cigomáticos: la técnica clásica de la ventana sinusal, descrita por Branemark en 1998, en la cual el implante hace un recorrido intrasinusal; la técnica quirúrgica simplificada, descrita por Stella \& Warner (2000), una variante de la técnica intrasinusal realizando una estrecha ranura sinusal y la técnica quirúrgica exteriorizada, descrita por Migliorança et al. (2006), que consiste en colocar los implantes cigomáticos extrasinusales, fijándolos a la pared lateral del seno y al hueso cigomático.
Aparicio et al. (2010), afirman que con la técnica quirúrgica exteriorizada se consigue una mayor estabilidad primaria, ya que el implante se fija a una mayor cantidad de cortical ósea, pero sólo es posible cuando existe una marcada concavidad vestibular en la región del seno. Concordamos con este autor porque la instalación de un implante extrasinusal no solo permite a los implantes fijarse a una mayor cantidad de cortical ósea, sino también elimina el riesgo de sinusitis.

Concluimos que existe importante diferencia entre los valores promedios encontrados para hombres y mujeres, dato relevante en la práctica clínica. Concluimos aún que el mayor espesor del hueso cigomático se encuentra en la zona media del hueso. Sugerimos que el anclaje superior del implante cigomático no debe extenderse hasta el margen posterosuperior del hueso cigomático, ya que el espesor del hueso a este nivel no ofrece condiciones de adecuada oseointegración de implantes.

\section{AGRADECIMIENTOS}

Al Departamento de Morfología y Genética de la Universidade Federal de São Paulo - UNIFESP, Brasil.

ALVES, N. Morphometric analysis of anatomic landmarks involved in surgical technique of zygomatic implants placement. Int. J. Morphol., 31(3):986-990, 2013.

SUMMARY: The aim of this study is to provide morphometric data of bony structures involved in the surgical technique of zygomatic implants, thus providing information to facilitate the planning and implementation of this procedure. Considering the average values obtained in our study, we conclude that the greatest thickness of the zygomatic bone is in the middle of the bone. We suggest that the upper anchorage of zygomatic implants should not extend to the posterior margin of the zygomatic bone, since the bone thickness at this level does not offer suitable conditions for osseointegration of implants.

KEY WORDS: Zygomatic implants; Morphometry.

\section{REFERENCIAS BIBLIOGRÁFICAS}

Alves, N. \& Cândido, P. L. Anatomia para o cirurgião-dentista. $2^{\text {a }}$ ed. São Paulo, Gen-Santos, 2013.

Aparicio, C.; Ouazzani, W.; Aparicio, A.; Fortes, V.; Muela, R; Pascual, A.; Codesal, M.; Barluenga, N.; Manresa, C. \& Franch, M. Extrasinus zygomatic implants: three year experience from a new surgical approach for patients with pronounced buccal concavities in the edentulous maxilla. Clin. Implant Dent. Relat. Res., 12(1):55-61, 2010.

Bedrossian, E.; Stumpel, L. 3rd.; Beckely, M. L. \& Indresano, T. The zygomatic implant: preliminary data on treatment of severely resorbed maxillae. A clinical report. Int. J. Oral Maxillofac. Implants, 17(6):861-5, 2002.

Bothur, S.; Jonsson, G. \& Sandahl, L. Modified technique using multiple zygomatic implants in reconstruction of the atrophic maxilla: a technical note. Int. J. Oral Maxillofac. Implants, 18(6):902-4, 2003.

Boyes-Varley, J. G.; Howes, D. G.; Lownie, J. F. \& Blackbeard, G. A. Surgical modifications to the Brånemark zygomaticus protocol in the treatment of the severely resorbed maxilla: a clinical report. Int. J. Oral Maxillofac. Implants, 18(2):232-7, 2003. 
Bränemark, P. I. Surgery and Fixture Installation. Zygomaticus Fixture Clinical Procedures. Sweden, Nobel Biocare AB, 1998.

Fernández Ateca, B.; Colorado Bonnin, N. \& Gay Escoda, C. Implantes transcigomáticos. Av. Periodon. Implantol., 16(3):12941, 2004.

Frodel, J. L. Jr.; Funk, G. F.; Capper, D. T.; Fridrich, K. L.; Blumer, J. R.; Haller, J. R. \& Hoffman, H. T. Osseointegrated implants: a comparative study of bone thickness in four vascularized bone flaps. Plast. Reconstr. Surg., 92(3):449-58, 1993.

González, E. D.; Sanz, R. A. \& Hidalgo, H. Estudio Anatómico en relación a los sitios comprometidos en la técnica de implantes cigomáticos. Int. J. Morphol., 25(1):133-7, 2007.

Migliorança, R. M.; Llg, J. P.; Serrano, A. S.; Souza, R.P. \& Zamperlini, M. S. Exteriorização de fixações zigomáticas em relação ao seio maxilar: uma nova abordagem cirúrgica. Implant News, 3(1):30-5, 2006.

Nakai, H.; Okazaki, Y. \& Ueda, M. Clinical application of zygomatic implants for rehabilitation of the severely resorbed maxilla: a clinical report. Int. J. Oral Maxillofac. Implants, 18(4):566-70, 2003.

Nkenke, E.; Hahn, M.; Lell, M.; Wiltfang, J; Schultze-Mosgau, S.; Stech, B.; Radespiel-Tröger, M. \& Neukam, F. W. Anatomic site evaluation of the zygomatic bone for dental implant placement. Clin. Oral Implants Res., 14(1):72-9, 2003.

Pi Urgell, J.; Revilla Gutiérrez, V. \& Gay Escoda, C. Rehabilitation of atrophic maxilla: A review of 101 zygomatic implants. Med. Oral Patol. Oral Cir. Bucal, 13(6):E363-70, 2008.

Stella, J. P. \& Warner, M. R. Sinus slot technique for simplification and improved orientation of zygomaticus dental implants: a technical note. Int. J. Oral Maxillofac. Implants, 15(6):88993, 2000 .

Stevenson, A. R. \& Austin, B. W. Zygomatic fixtures--the Sydney experience. Ann. R. Australas Coll. Dent. Surg., 15:337-9, 2000.

Tamura, H.; Sasaki, K. \& Watahiki R. Primary insertion of implants in the zygomatic bone following subtotal maxillectomy. Bull. Tokyo Dent. Coll., 41(1):21-4, 2000.

Uchida, Y.; Goto, M.; Katsuki, T. \& Akiyoshi T. Measurement of the maxilla and zygoma as an aid in installing zygomatic implants. J. Oral Maxillofac. Surg., 59(10):1193-8, 2001.

Vrielinck, L.; Politis C.; Schepers S.; Pauwels, M. \& Naert, I. Imagebased planning and clinical validation of zygoma and pterygoid implant placement in patients with severe bone atrophy using customized drill guides. Preliminary results from a prospective clinical follow-up study. Int. J. Oral Maxillofac. Surg., 32(1):714, 2003.
Weischer, T.; Schettler, D. \& Mohr, C. Titanium implants in the zygoma as retaining elements after hemimaxillectomy. Int. J. Oral Maxillofac. Implants, 12(2):211-4, 1997.

\author{
Dirección para correspondencia \\ Nilton Alves \\ Departamento de Odontología Integral Adultos \\ Facultad de Odontología \\ Universidad de La Frontera \\ Francisco Salazar, 1145, Temuco \\ CHILE
}

Email: niltonnalves@yahoo.com.br

Recibido : 05-05-2013

Aceptado: 19-06-2013 